\title{
差分电荷密度在电子结构分析中的教学实践
}

\author{
章佳菲 ${ }^{1}$, 白鸽 ${ }^{1}$, 徐余幸 ${ }^{1}$, 吴文清 $1,{ }^{*}$, 刘亚 ${ }^{1}$, 滕波涛 $1,2,{ }^{*}$ \\ 1 浙江师范大学化学与生命科学学院, 浙江 金华 321004 \\ 2 天津科技大学化工与材料学院, 天津 300457
}

\begin{abstract}
摘要: 差分电荷密度可以研究分子、团簇、固体材料以及分子与固体材料间相互作用导致的电荷重新分布, 广泛应 用于电子结构分析。作者在长期的理论研究与 “密度泛函理论在化学中的应用” 课程教学过程中发现, 差分电荷密 度计算根据研究对象和研究目标的不同, 计算方法与结果也大不相同。根据研究的目标, 差分电荷密度可以分为原 子基、碎片基、特定对象的差分电荷密度及自旋电荷密度。如果不能正确理解其概念, 选择合适的计算方法, 则 无法获取正确的电子结构信息。本文系统整理了常见与特殊差分电荷密度计算的类型、计算方法与适用研究体系, 通过课堂教师讲解与演示、学生练习, 结合探究性课后作业, 使学生深入理解、掌握这一重要电子分析方法, 为 教学与科研提供重要支持。
\end{abstract}

关键词：差分电荷密度；电子结构；密度泛函理论；自旋电荷密度图

中图分类号: G64; O6

\section{Teaching Practice of Charge Density Difference in Electronic Structure Analysis}

\author{
Jiafei Zhang ${ }^{1}$, Ge Bai ${ }^{1}$, Yuxing Xu ${ }^{1}$, Wenqing Wu 1,*, Ya Liu ${ }^{1}$, Botao Teng 1,2,* \\ ${ }^{1}$ College of Chemistry and Life Sciences, Zhejiang Normal University, Jinhua 321004, Zhejiang Province, China. \\ ${ }^{2}$ College of Chemical and Materials Science, Tianjin University of Science \& Technology, Tianjin 300457, China.
}

\begin{abstract}
Charge density difference (CDD) can investigate the charge redistribution of molecules, clusters and solid materials, as well as the interaction between molecules and solid materials, which has been widely used in electronic structure analysis. Based on the authors' theoretical research and teaching practice of "application of density functional theory in chemistry research", the authors found that the calculation methods and results of charge density difference vary along with the different research objectives. According to the research objectives, the charge density difference can be divided into atom-based, fragment-based, specific object-based and spin density. If one can't understand the concept correctly and establish a reasonable model, it is hard to get the correct electronic structure information. In the present work, authors systematically sort out the types, calculation methods and applicable research system of CDD calculation. Base on the teacher's explanation and demonstration, students' practice, as well as exploratory homework, students can understand and master the electronic analysis method of charge density difference, which provides important support for teaching and scientific research.
\end{abstract}

Key Words: Charge density difference; Electronic structure; Density functional theory; Spin density

收稿: 2021-07-31; 录用: 2021-09-16; 网络发表: 2021-10-19

“通讯作者, Emails: wuwenqing1985@126.com (吴文清); tbt@zjnu.cn (滕波涛)

基金资助：国家自然科学基金(21872125); 浙江省教改项目(jg20180064) 
基于第一性原理的理论计算可以从分子、原子水平研究物质的结构, 并从微观电子角度研究原 子、分子、团簇、固体材料等的电子结构, 探究材料的结构-性能间的构效关系, 为材料的改性及新 材料的开发提供理论支撑。以催化材料研究与开发为例, 通过理论计算催化剂的电子结构及反应物 与催化剂间相互作用, 认识催化反应的活性中心与活性物种, 进而研究催化反应机理, 建立可能的 催化剂结构与性能的关联, 为催化剂的理性设计提供重要依据 ${ }^{[1]}$ 。随着计算化学与高性能计算机的 迅速发展, 基于第一性原理的理论计算在化学与材料研究中广泛应用, 很多高校为研究生及高年级 本科生开设了相关的课程 ${ }^{2-4]}$, 其中电子结构分析是教学的重点之一。

在众多电子结构分析方法中, 电荷可以获得体系中每个原子相比孤立原子的电子得失情况; 静 电势可反映体系不同位置的亲电与亲核性; 电荷密度反映了体系中电子分布情况; 能带结构与态密 度可以获取材料在价带、导带等的电子结构; 分子轨道可以获取电子在分子不同能级轨道中的分布。 与以上电子结构分析方法不同, 差分电荷密度可以研究分子、团簇、固体材料、分子与固体材料间 相互作用导致的电荷重新分布, 因此广泛应用于电子结构分析 ${ }^{[5]}$ 。作者在长期的理论计算研究与 “密 度泛函理论在化学中的应用” 课程教学过程中发现, 由于差分电荷密度计算要根据研究对象和研究 目标的不同, 具体计算过程不同, 计算的结果也大不相同。如果不能正确理解其概念, 选择合适的 计算方法, 则无法获取正确的电子结构信息。因此, 差分电荷密度计算是电子结构分析教学的难点 和重点。本文系统整理了常见与特殊差分电荷密度计算的类型、计算方法与适用研究体系, 通过课 堂教师讲解与演示、学生练习, 结合探究性课后作业, 使学生深入理解、掌握这一重要电子分析方 法的概念、目标与计算方法, 为相应教学与科研提供重要信息。

\section{1 差分电荷密度定义、计算与讨论}

差分电荷密度主要考查研究对象在相互作用中电子的移动与再分布情况, 按照差分电荷密度计 算方法的不同, 差分电荷密度可以分为原子基、碎片基与特定对象的差分电荷密度以及自旋电荷密 度。计算差分电荷密度的程序有很多, 本文给出的例子均是采用Vienna ab initio simulation package (VASP)计算的, 差分电荷密度图由VESTA软件获得。下面分别对不同类型的差分电荷密度进行讨论 分析。

\section{1 原子基差分电荷密度}

原子基差分电荷密度是指研究对象(分子、团簇或模型催化剂)成键后的电子云密度与组成其结 构的原子电子云密度之差。例如, 分子 $\mathrm{AB}$ 由原子 $\mathrm{A}$ 与 $\mathrm{B}$ 组成, 其差分电荷密度为分子 $\mathrm{AB}$ 的电子云密 度分布减去原子 $\mathrm{A}$ 与 $\mathrm{B}$ 的电子云密度, 计算公式如下:

$$
\Delta \rho=\rho_{\mathrm{AB}}-\rho_{\mathrm{A}}-\rho_{\mathrm{B}}
$$

其中 $\rho_{\mathrm{AB}} 、 \rho_{\mathrm{A}}$ 与 $\rho_{\mathrm{B}}$ 分别为分子 $\mathrm{AB}$ 、原子 $\mathrm{A}$ 与 $\mathrm{B}$ 的电子云密度。若研究对象为多原子分子、团簇或 模型催化剂, 其差分电荷密度为系统的总电子云密度减去组成其结构的各原子电子云密度之和, 计 算公式为:

$$
\Delta \rho=\rho_{\text {total }}-\sum \rho_{i}
$$

其中 $\rho_{\mathrm{total}}$ 与 $\rho_{i}$ 分别为总电子云密度与 $i$ 原子的电子云密度。

原子基差分电荷密度可得到研究对象(分子、团簇或催化剂)成键和成键电子耦合过程中的电子 移动与成键极化方向等性质, 多用于分析分子、团簇成键情况。

图1分别给出了 $\mathrm{CO}_{2} 、 \mathrm{O}_{2}$ 、四层 $\mathrm{Pd}(111)$ 表面原子基差分电荷密度图。由图1(a)可以看出, 当 $\mathrm{C}$ 与 $\mathrm{O}$ 原子相互作用形成 $\mathrm{CO}_{2}$ 时, 电子发生了重新排布, $\mathrm{C}$ 原子以失电子为主(蓝色), $\mathrm{O}$ 原子以得电子为主 (黄色), 电子在 $\mathrm{C}$ 与 $\mathrm{O}$ 原子间重新分布形成了 $\mathrm{C}-\mathrm{O}$ 共价键; 同时 $\mathrm{CO}_{2}$ 分子得失电子情况上下对称, 是非 极性分子。 $\mathrm{O}_{2}$ 为同核双原子分子, $\mathrm{O}$ 原子在得失电子的同时, $\mathrm{O}-\mathrm{O}$ 间形成较强相互作用的共价键, 如图1(b)所示。同时由于同核双原子分子对称, $\mathrm{O}-\mathrm{O}$ 键没有极性, 是非极性分子。由图1(c)的 $\mathrm{Pt}(111)$ 四层周期性模型的原子基差分电荷密度图可以看出, 电子在 $\operatorname{Pt}(111)$ 模型的上下表面略有富集, 而中 间两层以失电子为主，这一结果也可由相应的电荷分析进一步证明。 
(a)

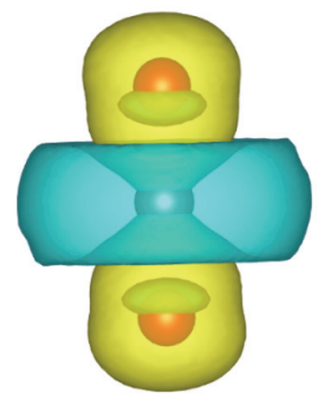

(b)

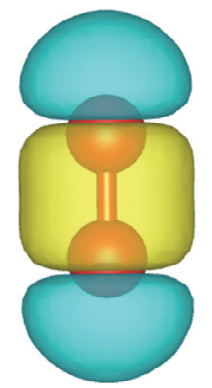

(c)

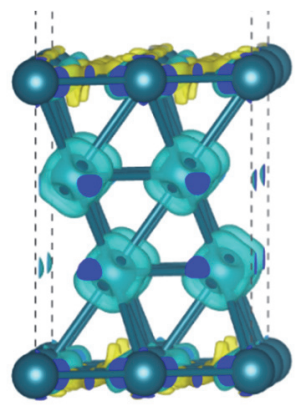

图1 $\mathrm{CO}_{2}(\mathrm{a}) 、 \mathrm{O}_{2}(\mathrm{~b})$ 与 $\mathrm{Pd}(111)(\mathrm{c})$ 的原子基差分电荷密度图

红色、黑色、深蓝色球分别为 $\mathrm{O} 、 \mathrm{C} 、 \mathrm{Pd}$ 原子, 蓝色为失电子, 黄色为得电子; (a) 与 $(\mathrm{b})$ 等值面为 $0.003 \mathrm{e} \cdot \mathrm{a}_{0}^{-3}$; 图 (c) 为 $0.005 \mathrm{e} \cdot \mathrm{a}_{0}^{-3}$ 计算参数设置: 截止能为 $400 \mathrm{eV}$, 自洽场能量收玫标准为 $10^{-5} \mathrm{eV}$, 力的收玫标准为 $0.2 \mathrm{eV} \cdot \mathrm{nm}^{-1}, \operatorname{Pd}(111)$ 的 $k$ 点为 $5 \times 5 \times 1$ 电子版为彩图

\section{2 碎片基差分电荷密度}

碎片基差分电荷密度定义: 体系总电荷密度减去吸附分子与基底(或模型催化剂)的电荷密度, 计算公式如下:

$$
\Delta \rho=\rho_{\mathrm{A} / \mathrm{S}}-\rho_{\mathrm{A}}-\rho_{\mathrm{S}}
$$

其中 $\rho_{\mathrm{A} / \mathrm{S}}$ 为吸附分子与基底的总电荷密度, $\rho_{\mathrm{A}}$ 和 $\rho_{\mathrm{S}}$ 分别为吸附分子与基底(或模型催化剂)的电荷 密度。通过碎片基差分电荷密度的计算和分析, 可以分析吸附分子与基底(或模型催化剂)间的电子 相互作用。

图2给出了 $\mathrm{CF}_{3} \mathrm{CH}_{2} \mathrm{I}$ 在 $\mathrm{Ag}(111)$ 表面吸附时 $\mathrm{CF}_{3} \mathrm{CH}_{2} \mathrm{I}$ 与接触的 $\mathrm{Ag}$ 原子相互作用的差分电荷密度 图。由图中可以看出, 当等值面取值为 $0.01 \mathrm{e}^{-3} \mathrm{a}_{0}^{-3}$ 时, 几乎看不到两者的电子相互作用; 当等值面取 $0.001 \mathrm{e}^{-} \mathrm{a}_{0}^{-3}$ 时, 两者电子相互作用比较明显, 即 $\mathrm{Ag}$ 失电子(蓝色), $\mathrm{CF}_{3} \mathrm{CH}_{2} \mathrm{I}$ 的最高占据轨道 $(\mathrm{HOMO}) n_{\mathrm{I}}$ 轨道(未成键 $p$ 电子)失电子, 其最低未占据轨道 $(\mathrm{LUMO}) \sigma_{\mathrm{C}-\mathrm{I}}{ }^{*}$ 得电子(黄色), $\mathrm{Ag}$ 与 $\mathrm{I}$ 原子之间形成一定 的相互作用, 如图2(b)中 $\mathrm{Ag}-\mathrm{I}$ 之间的黄色部分所示。差分电荷密度直观清晰地表明了吸附分子与模 型催化剂 $\mathrm{Ag}(111)$ 表面的电子相互作用。从差分电荷密度不同的等值面也可以看出, $\mathrm{CF}_{3} \mathrm{CH}_{2} \mathrm{I}$ 与 $\mathrm{Ag}$ 有 一定相互作用, 但不是很强, 这与其计算的吸附能为 $-0.27 \mathrm{eV}$ 相一致 ${ }^{[6]}$ 。

(a)

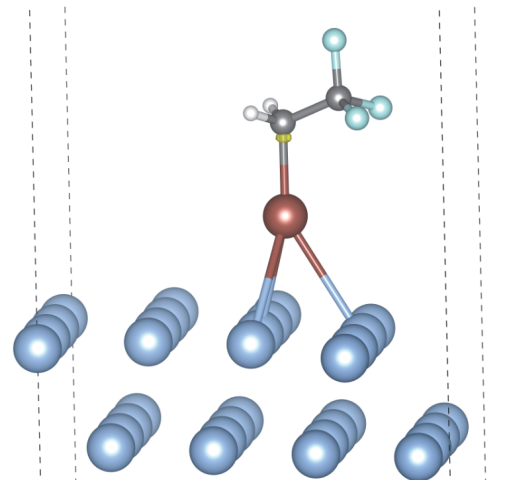

(b)

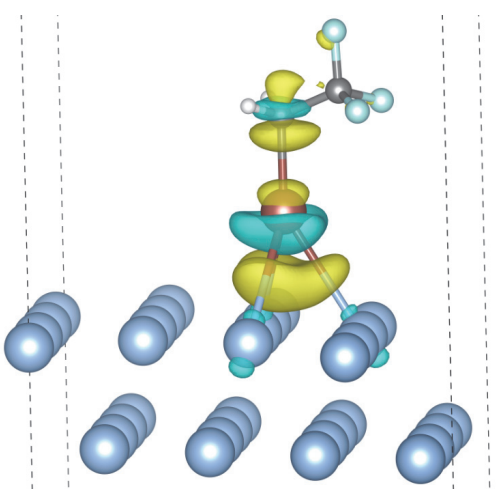

图2 $\mathrm{CF}_{3} \mathrm{CH}_{2} \mathrm{I} / \mathrm{Ag}(111)$ 表面的差分电荷密度图

蓝色、淡蓝色、黑色、灰色、棕色球分别为 $A g 、 F 、 C 、 H 、 I$ 原子; 等值面为 0.01 (a) 与 $0.001 \mathrm{e}^{-3} \mathrm{a}_{0}^{-3}(\mathrm{~b})$; 计算参数设置: 截止能为 $400 \mathrm{eV}$, 自洽场能量收玫标准为 $10^{-5} \mathrm{eV}$, 力的收玫标准为 $0.2 \mathrm{eV} \cdot \mathrm{nm}^{-1}, k$ 点为 $3 \times 3 \times 1$ 电子版为彩图

与 $\mathrm{CF}_{3} \mathrm{CH}_{2} \mathrm{I}$ 不同, 当 $\mathrm{CF}_{3} \mathrm{CH}_{2}$ 与 $\mathrm{Ag}(111)$ 相互作用时, 由图3不同等值面的差分电荷密度图可以看 出, 当等值面取 $0.01 \mathrm{e} \cdot \mathrm{a}_{0}^{-3}$ 时, 两者电子相互作用就很明显, $\mathrm{Ag}$ 失电子, $\mathrm{CH}_{2} \mathrm{CF}_{3}$ 得电子; 当等值面取 $0.001 \mathrm{e} \cdot \mathrm{a}_{0}^{-3}$ 时, 两者强电子作用更明显, 这与其较强的吸附能 $(-1.57 \mathrm{eV})$ 相一致。 
(a)

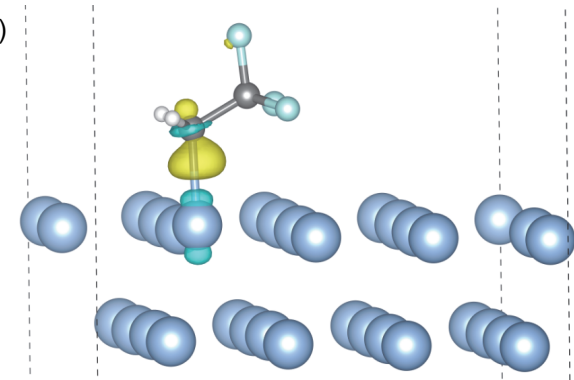

(b)

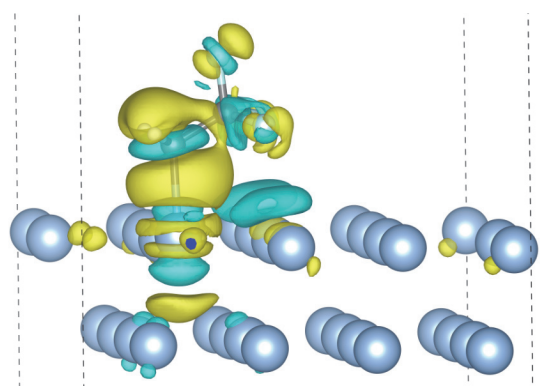

图3 $\mathrm{CF}_{3} \mathrm{CH}_{2} / \mathrm{Ag}(111)$ 表面的差分电荷密度图

等值面为 $0.01(\mathrm{a})$ 与 $0.001 \mathrm{e} \cdot \mathrm{a}_{0}^{-3}(\mathrm{~b})$, 计算参数设置同图2

基于碎片基的差分电荷密度还可研究金属纳米粒子与氧化物载体间的电子转移与分布情况。图4 分别给出了金团簇 $\mathrm{Au}_{3} 、 \mathrm{Au}_{9}$ 与 $\mathrm{CeO}_{2}(111)$ 表面相互作用时的电子转移情况。由图4可以看出, 当 $\mathrm{Au}_{3}$ 与 $\mathrm{Au}_{9}$ 团簇与氧化铈相互作用时, 金团簇中与氧化铈载体接触的金原子失电子 (金原子中蓝色部分), 氧 化铈载体中的 $\mathrm{Ce}^{4+}$ 离子得电子, 形成 $\mathrm{Ce}^{3+}$ (铈离子中黄色部分)。同时还可以发现, $\mathrm{Au}_{9}$ 团簇与氧化铈 载体相互作用, 主要是金团簇底部与氧化铈接触的原子失电子 ${ }^{[7]}$ 。因此, 差分电荷密度可以直观地 得到金属纳米粒子与氧化物载体相互作用产生的电子转移与分布。

(a)

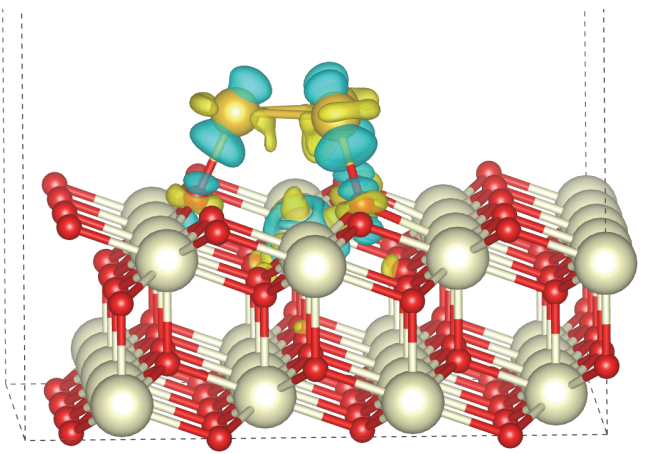

(b)

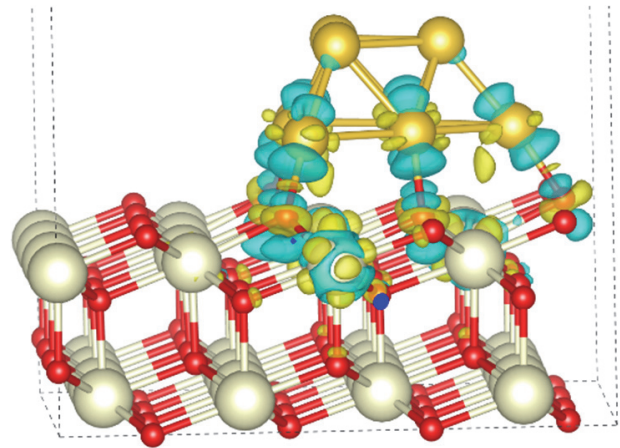

图4 $\mathrm{Au}_{3}(\mathrm{a}) 、 \mathrm{Au} 9 \mathrm{(b)}$ 与 $\mathrm{CeO}_{2}(111)$ 表面相互作用的差分电荷密度图 黄色、红色、金色球为 $\mathrm{Ce} 、 \mathrm{O} 、 \mathrm{Au}$ 原子，等值面为 $0.005 \mathrm{e} \cdot \mathrm{a}_{0}^{-3}$;

计算参数设置: 截止能为 $400 \mathrm{eV}$, 自洽场能量收玫标准为 $10^{-5} \mathrm{eV}$, 力的收玫标准为 $0.5 \mathrm{eV} \cdot \mathrm{nm}^{-1}, k$ 点为 $2 \times 2 \times 1$ 电子版为彩图

\section{3 自旋电荷密度}

自旋电荷密度是特殊的差分电荷密度, 是体系自旋向上的电荷密度减去自旋向下电荷密度, 具 体计算公式如下:

$$
\Delta \rho=\rho_{\text {up }}-\rho_{\text {down }}
$$

其中 $\rho_{\text {up }}$ 与 $\rho_{\text {down }}$ 分别为自旋向上与自旋向下的电子云密度。

自旋电荷密度通常用来研究体系(分子、团簇、模型催化剂)的自旋性质 ${ }^{[8]}$ 。以催化氧化反应中非 常关注的氧气、超氧、过氧等氧物种为例, 由于 $\mathrm{O}_{2}$ 分子的最稳定结构为三重态, 分子中两个自旋单 电子分别填充于 2 个 $2 \pi^{*}$ 轨道, 其自旋电荷密度图为哑铃形, 如图5(b)上方图所示; 当其得到一个电子 形成超氧物种 $\mathrm{O}_{2}^{-}$, 只有一个自旋电子在 $2 \pi^{*}$ 轨道, 如图 5 (a) 所示, 其自旋电荷密度图为蝴蝶形, 如 图5(b)中间图所示; 当其得到两个电子形成超氧物种 $\mathrm{O}_{2}^{2-}, 2 \pi^{*}$ 没有自旋电子, 自旋电荷密度图看不到 自旋电子, 如图 5(b)下方图所示。因此, 可以根据图5(b)中的自旋电荷密度图的分子氧、超氧与过氧 的自旋电子结构作为不同氧物种的识别依据。 
(a)

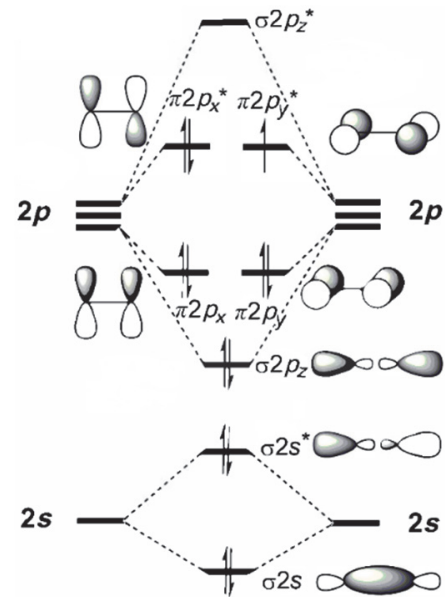

(b)
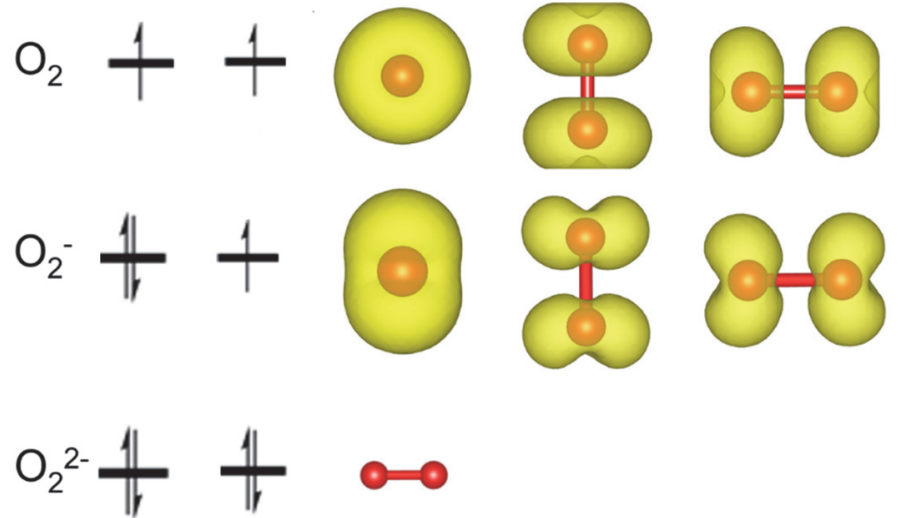

图5 不同氧物种电子排布与自旋电荷密度图

(a) $\mathrm{O}_{2}^{-}$分子轨道示意图; (b) $\mathrm{O}_{2} 、 \mathrm{O}_{2}^{-}$与 $\mathrm{O}_{2}^{2-}$ 的自旋电荷密度图

图4的碎片基的差分电荷密度图结果表明, 金团簇负载在氧化铈载体上, 由于金团簇与载体相互 作用产生的电子转移金团簇失电子, 氧化铈中的Ce离子得电子。但是, 由于碎片基差分电荷密度图 中显示金团簇与氧化铈各有电子得失, $\mathrm{Ce}$ 离子得电子情况并不直观。由于 $\mathrm{Ce}$ 原子外层电子排布为 $5 s^{2} 5 p^{6} 4 f^{1} 5 d^{1} 6 s^{2}$, 当其为 $\mathrm{Ce}^{4+}$ 离子时, 没有自旋电子, 当其得一个电子生成 $\mathrm{Ce}^{3+}$ 离子时, 在 $f$ 轨道填充 一个自旋电子, 因此在自旋密度图中表现出 $f$ 轨道特有的轨道形状。图6所示的 $\mathrm{Au}_{x} / \mathrm{CeO}_{2}(111)$ 的自旋 电荷密度图可以清晰地看到, $\mathrm{Au}_{3} / \mathrm{CeO}_{2}(111)$ 中 $\mathrm{Au}_{3}$ 与氧化铈相互作用, $\mathrm{Au}_{3}$ 上的电子转移到一个 $\mathrm{Ce}^{4+}$ 上, 形成一个 $\mathrm{Ce}^{3+}$ 离子, 而 $\mathrm{Au}_{9} / \mathrm{CeO}_{2}(111)$ 由于有较多 $\mathrm{Au}$ 原子与载体相互作用, 导致 3 个电子转移到氧 化铈载体上, 形成 3 个 $\mathrm{Ce}^{3+}$ 离子。

(a)

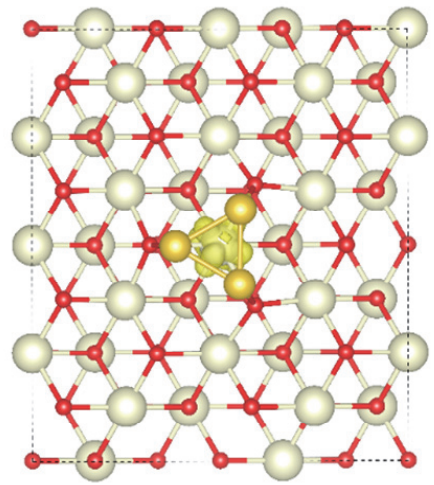

(b)

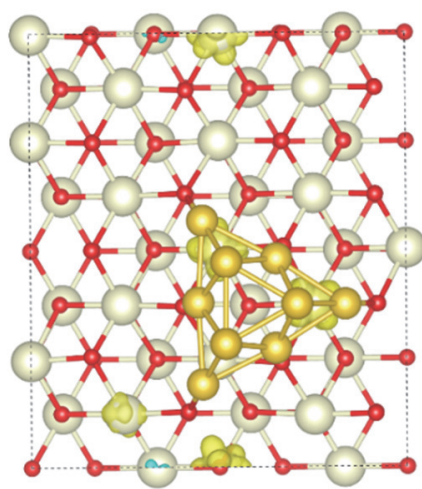

图6 $\mathrm{Au}_{3} / \mathrm{CeO}_{2}(111)(\mathrm{a})$ 与 $\mathrm{Au}_{9} / \mathrm{CeO}_{2}(111)(\mathrm{b})$ 的自旋电荷密度图 等值面为 $0.005 \mathrm{e} \cdot \mathrm{a}_{0}^{-3}$, 计算参数设置同图 4

自旋电荷密度还可以研究吸附物种与载体相互作用发生的电子转移。如图7(b)所示, 当氧化铈 载体表面失去一个氧原子形成一个氧缺陷时, 在氧化铈载体表面留下两个电子, 被两个 $\mathrm{Ce}^{4+}$ 离子获 取形成两个 $\mathrm{Ce}^{3+}$ 离子, 可以由图7(b)看出载体表面有两个 $4 f$ 轨道的自旋电子。当 $\mathrm{O}_{2}$ 分子吸附于其中一 个 $\mathrm{Ce}^{3+}$ 离子上, 其中 4 轨道上的 1 个电子转移至 $\mathrm{O}_{2}$ 上形成时 $\mathrm{O}_{2}^{-}$, 具有超氧自旋电荷密度图的特征, 同 时 $\mathrm{Ce}^{3+}$ 离子失电子再次变成 $\mathrm{Ce}^{4+}$, 如图7(a)所示。当 $\mathrm{O}_{2}$ 分子吸附于表面氧缺陷时, 载体上两个 $4 f$ 轨道 自旋电子都转移至 $\mathrm{O}_{2}$ 上形成时 $\mathrm{O}_{2}^{2-}$, 载体与超氧均没有自旋电子, 即两个 $\mathrm{Ce}^{3+}$ 离子均被氧化为 $\mathrm{Ce}^{4+}$, 如图7(c)所示。因此, 自旋电荷密度图清晰、直观地给出了表面吸附物种与载体相互作用产生的电子 转移情况 ${ }^{[9]}$ 。 
(a)

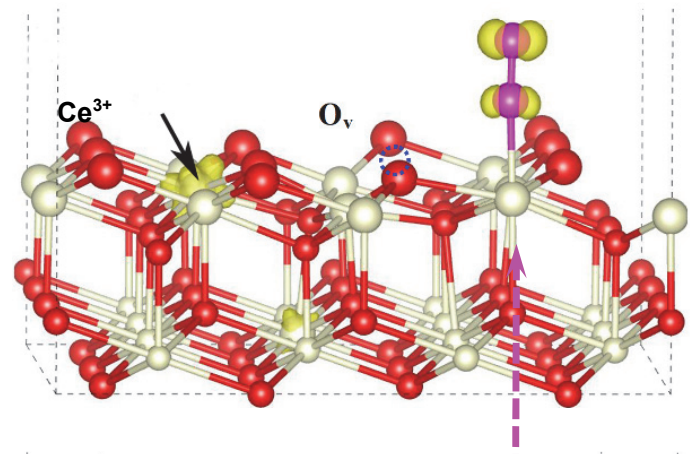

(b)

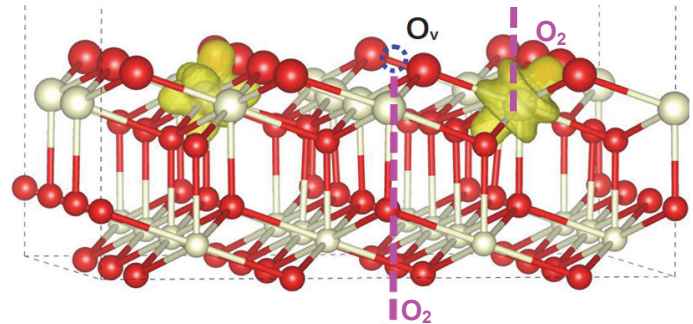

(c)

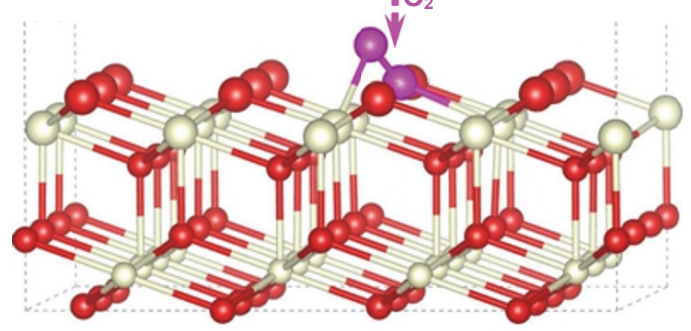

图7 $\mathrm{CeO}_{2}(111)$ 表面超氧与过氧物种形成过程

红色与水红色球分别为 $\mathrm{CeO}_{2}$ 与 $\mathrm{O}_{2}$ 中 $\mathrm{O}$ 原子, 等值面为 $0.005 \mathrm{e}^{-3} \mathrm{a}_{0}^{-3}$, 计算参数设置同图 4 电子版为彩图

\section{4 特定对象的差分电荷密度}

特定对象的差分电荷密度研究是根据研究体系与目标的不同, 无法由原子基或碎片基进行差分 电荷密度的计算, 需要根据研究体系的特点及研究目标不同, 选择特定的电荷密度差分背景, 其计 算公式可以用下式表示:

$$
\Delta \rho=\rho_{\mathrm{A}}-\rho_{\mathrm{B}}
$$

其中 $\rho_{\mathrm{A}}$ 与 $\rho_{\mathrm{B}}$ 分别为研究对象与特定差分对象的电荷密度。

众所周知, 由于金属团簇面、棱、角上的原子配位不饱和度较高, 可以形成电子富集, 导致其 电荷密度较内部原子高。如图 $8(\mathrm{a})$ 为 $\mathrm{Pd}_{35}$ 团簇的电荷密度图, 由于团簇内部金属与表面和棱角上的电 子云密度相差不是特别大, 从团簇的电荷密度图8(a)中很难看出电子在面、棱及角上发生富集。 图8(b)为基于原子基的差分电荷密度图, 其计算方式为团簇的电荷密度减去各孤立Pd金属原子的电 荷密度。因此, 在部分观察到电子云在团簇的角、棱富集的同时, 主要体现了电子云在两个相邻Pd 原子间的富集，表明Pd金属间形成了金属键。

为清晰获取电子在 $\mathrm{Pd}_{35}$ 团簇的面、棱与角上的富集，可以将体相 $\mathrm{Pd}$ 金属形成的电荷密度作为背 景, 用 Pd团簇的电荷密度减去体相 $\mathrm{Pd}$ 金属的电荷密度, 如图8(c)所示。其中, 体相 $\mathrm{Pd}$ 金属的电荷密度 由相同格子大小的体相Pd金属超晶胞计算。图8(c)可以看出, 电子在金属团簇的面、棱、角的富集程 度依次增加, 同时图8(c)中观察不到金属间相互作用形成的金属键电子, 因此可以清晰、直观地展示 金属团簇电子云富集情况，为解释金属催化剂面、棱、角不同反应性能提供了一定的理论依据。 
(a)

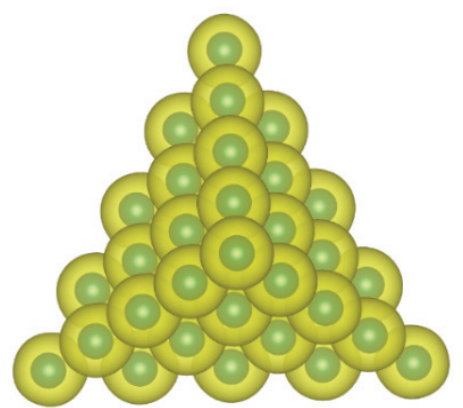

(b)

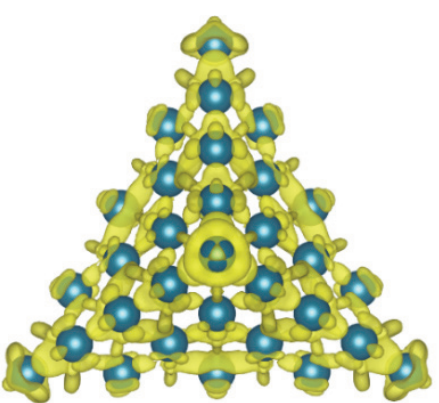

(c)

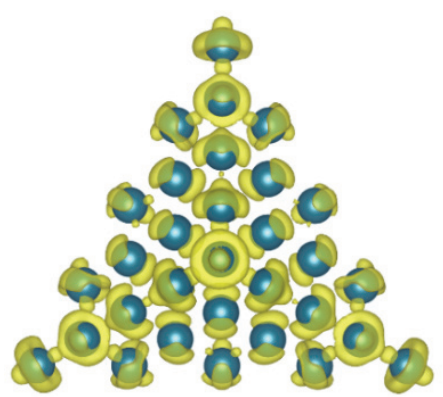

图8 Pd 35 团簇的电荷密度图(a)、基于原子(b)与体相金属(c)电子分布的差分电荷密度图

(a) 等值面为 $0.05 \mathrm{e} \cdot \mathrm{a}_{0}^{-3}$, (b)与 (c)均为 $0.004 \mathrm{e} \cdot \mathrm{a}_{0}^{-3}$

计算参数设置: 截止能为 $400 \mathrm{eV}$, 自洽场能量收玫标准为 $10^{-5} \mathrm{eV}$, 力的收玫标准为 $0.2 \mathrm{eV} \cdot \mathrm{nm}^{-1}$

综上, 通过教师课堂的系统讲解与分析, 使学生掌握以下四点: (1) 原子基差分电荷密度是以各 孤立原子自身电荷密度的和为差分背景的, 可得到研究对象成键和成键电子耦合过程中的电子移动 与成键极化方向等性质; (2) 碎片基的差分电荷密度以吸附分子与碎片的电荷密度为差分背景, 可 获得吸附物种与吸附剂间的电子转移与分布情况, 即电子转移主要发生在哪些原子之间, 转移电子 的数量, 进而初步判断出两者的相互作用强弱; (3) 自旋电荷密度是体系自旋向上的电荷密度减去 自旋向下电荷密度, 可获得磁性体系中自旋电子分布、传递及特征物种识别等信息; (4) 特定对象的 差分电荷密度是根据具体的研究目标, 选择特定的电荷密度背景差分, 获取差分电荷密度信息, 进 而分析电子结构, 为解释特定化学结构、反应性能等提供微观电子解释。

\section{2 结语}

通过课堂教师对基本概念的讲解, 简单实例的计算、作图演示与学生的当堂练习, 激发了学生 的学习兴趣, 加深了学生对概念的理解。结合课后探究性作业, 学生能够深入理解原子基与碎片基 差分电荷密度、自旋电荷密度及特定对象的差分电荷密度的概念, 并与结构化学中的原子、双原子 分子、多原子分子的结构和性质, 配位化合物、金属的结构和性质相关知识关联, 初步掌握根据不 同研究目标, 选择合适计算方法, 开展相应计算, 获取电子结构信息, 助力其科研工作; 同时培养 学生具体问题具体分析, 进而解决问题的能力。

\section{参 考 文 献}

[1] 周公度, 段连运. 结构化学基础. 第3版. 北京: 北京大学出版社, 2002: 53-60.

[2] 谢坚坚, 滕波涛, 楼怡林, 赵雷洪. 化学教育, 2017, 38 (12), 66 .

[3] 滕波涛, 谢坚坚, 冯姗, 刘亚. 化学教育, 2016, 37 (22), 57.

[4] 刘亚，曾贤璐，胡釒釙，蒋永福，代伟，郝庆兰，章康达，滕波涛. 化学教育(中英文), 2018, 39 (18), 20.

[5] Zhu, K. J.; Wang, F.; Teng, B. T.; Wen, X. D.; Fan, M. H.; Liu, X. N. J. Colloid Interface Sci. 2018, 512, 775.

[6] Teng, B. T.; Zhao, Y.; Wu, F. M.; Wen, X. D.; Chen, X. P.; Huang, W. X. Surf. Sci. 2012, 606 (15-16), 1227.

[7] Teng, B. T.; Lang, J. J.; Wen, X. D.; Zhang, C.; Fan, M. H. J. Phys. Chem. C 2013, 117 (37), 18986.

[8] 周玉芬, 杨艳菊, 滕波涛. 大学化学, 2017, 32 (10), 61.

[9] Zhao, Y.; Teng, B. T.; Wen, X. D.; Zhao, Y.; Chen, Q. P.; Zhao, L. H.; Luo, M. F. J. Phys. Chem. C 2012, 116 (30), 15986. 